\title{
ELEMENTARY METHODS IN THE THEORY OF PRIMES
}

\author{
BY \\ PATRICK R. AHERN( $\left.{ }^{1}\right)$
}

Introduction. To facilitate the discussion of elementary formulas in the first section, we introduce the ring of formal Dirichlet series on the integral ideals of an algebraic number field. We show how formal Dirichlet series can be employed systematically to give proofs of some well-known results in elementary number theory. Then we give a new proof of Selberg's fundamental formula for algebraic number fields and a very simple proof of a somewhat stronger result first proved by Amitsur using his symbolic calculus. Next we consider formal Dirichlet series involving characters of ideal classes modulo an integral ideal $\mathrm{m}$, and obtain several new asymptotic formulas involving these characters. Using these formulas we give a new proof of Selberg's formula for ideals in ideal classes and derive a new result standing in the same relation to Selberg's formula for ideal classes as Amitsur's result is to Selberg's formula for number fields.

In the second section, motivated by some results in the first section, we are led to consider certain Tauberian theorems in order to get a new proof of the equidistribution of prime ideals in ideal classes. Certainly these methods yield neither the most elegant nor the most elementary proof of this result but the methods may be of some interest in themselves. We are led to consider Tauberian theorems involving complex-valued functions where previously only real-valued functions had been considered and we show our results are, in some sense, best possible. We also consider the situation where we have several functions satisfying simultaneous and interdependent Tauberian conditions.

\section{Elementary formulas.}

Definition 1.1. Let $k$ be an algebraic number field with ring of integers $R$, then the ring $D$ of formal Dirichlet series on $k$ is the set of complex-valued functions defined on the set $I$ of integral ideals of $k$ with the following operations:

(a) For $\alpha, \beta \in D$ and $\mathfrak{a} \in I ;(\alpha+\beta)(\mathfrak{a})=\alpha(\mathfrak{a})+\beta(\mathfrak{a})$.

(b) For $\alpha, \beta \in D$ and $\mathfrak{a} \in I ;(\alpha \beta)(\mathfrak{a})=\Sigma_{\mathfrak{b} \supseteq \mathfrak{a}} \alpha(\mathfrak{a}) \beta(\mathfrak{a b}-1)$.

It is easily seen that with these operations $D$ becomes a commutative ring with identity $e$, where $e(R)=1$ and $e(\mathfrak{a})=0$ for $\mathfrak{a} \neq R$. Furthermore $\alpha \in D$ has an inverse if and only if $\alpha(R) \neq 0$.

Received by the editors September 30, 1963. 18918.

${ }^{1}{ }^{1}$ This research was partially supported by National Science Foundation Grant G- 
DEFINITION 1.2.

(a) We say $\alpha \in D$ is strongly multiplicative if $\alpha(R)=1$ and $\alpha(\mathfrak{a} \mathfrak{b})=\alpha(\mathfrak{a}) \alpha(\mathfrak{b})$ for all $\mathfrak{a}, \mathfrak{b} \in I$.

(b) We define $\zeta \in D$ by $\zeta(\mathfrak{a})=1$ for all $\mathfrak{a} \in I$.

(c) We define $\mu(\mathfrak{a})=(-1)^{r}$ if $\mathfrak{a}=\mathfrak{p}_{1} \cdots \mathfrak{p}_{r}$ where $\mathfrak{p}_{1}, \cdots, \mathfrak{p}_{r}$ are distinct prime ideals, and $\mu(\mathfrak{a})=0$ otherwise.

(d) If $\alpha \in D$ we define $\alpha^{\prime} \in D$ by $\alpha^{\prime}(\mathfrak{a})=-\alpha(\mathfrak{a}) \log N a$ for all $\mathfrak{a} \in I$, where $N \mathfrak{a}$ denotes the norm of $\mathfrak{a}$.

(e) For $\alpha \in D, x \geqq 0$, let $C(\alpha, x)=\Sigma_{N_{\mathfrak{a}} \leqq x} \alpha(\mathfrak{a})$.

(f) We define $\Lambda(\mathfrak{a})=\log N(\mathfrak{a})$ if $\mathfrak{a}=\mathfrak{p}^{n}$ for some prime ideal $\mathfrak{p}$ and $\Lambda(\mathfrak{a})=0$ otherwise.

Using the above definitions and unique factorization for ideals in algebraic number fields, it is straightforward to verify the following:

(1.1) $\log N \mathfrak{a}=\Sigma_{\mathfrak{b} \supseteq \mathfrak{a}} \Lambda(\mathfrak{b})$.

(1.2) For $\alpha, \beta \in D,(\alpha+\beta)^{\prime}=\alpha^{\prime}+\beta^{\prime}$ and $(\alpha \beta)^{\prime}=\alpha^{\prime} \beta+\alpha \beta^{\prime}$.

(1.3) If $\alpha \in D$ and $\alpha(R) \neq 0$, then $\left(\beta \alpha^{-1}\right)^{\prime}=\left(\alpha \beta^{\prime}-\beta \alpha^{\prime}\right)\left(\alpha^{-1}\right)^{2}$.

(1.4) For all $\mathfrak{a} \in I, \zeta^{-1}(\mathfrak{a})=\mu(\mathfrak{a})$.

(1.5) If $\alpha \in D$ is strongly multiplicative then $\alpha^{-1}(\mathfrak{a})=\mu(\mathfrak{a}) \alpha(\mathfrak{a})$ for all $\mathfrak{a} \in I$.

(1.6) If $\alpha \in D$ is strongly multiplicative then $-\alpha^{\prime} \alpha^{-1}(\mathfrak{a})=\Lambda(\mathfrak{a}) \alpha(\mathfrak{a})$ for all $a \in I$.

(1.7) For $\alpha, \beta \in D$ we have

$$
C(\alpha \beta, x)=\sum_{N \mathfrak{a} \leqq x} \alpha(\mathfrak{a}) C\left(\beta, \frac{x}{N \mathfrak{a}}\right)=\sum_{N \mathfrak{a} \leqq x} \beta(\mathfrak{a}) C\left(\alpha, \frac{x}{N \mathfrak{a}}\right) .
$$

Next we prove an identity from which we will be able to deduce several asymptotic formulas.

THEOREM 1.1. Let $\alpha, \phi \in D$ with $\alpha$ strongly multiplicative; then

$$
\begin{aligned}
C(\phi, x) \log x & +\sum_{N \mathfrak{a} \leqq x} \alpha(\mathfrak{a}) \Lambda(\mathfrak{a}) C\left(\phi, \frac{x}{N \mathfrak{a}}\right) \\
& =\sum_{N \mathfrak{a} \leqq x} \mu(\mathfrak{a}) \alpha(\mathfrak{a}) \log \frac{x}{N \mathfrak{a}} C\left(\phi \alpha, \frac{x}{N \mathfrak{a}}\right) .
\end{aligned}
$$

Proof. Consider the expression

$$
\begin{aligned}
\sum_{N \mathfrak{a} \leqq x} \mu(\mathfrak{a}) \alpha(\mathfrak{a}) \log & \frac{x}{N \mathfrak{a}} C\left(\phi \alpha, \frac{x}{N \mathfrak{a}}\right) \\
= & \log x \sum_{N_{\mathfrak{a}} \leqq x} \mu(\mathfrak{a}) \alpha(\mathfrak{a}) C\left(\phi \alpha, \frac{x}{N \mathfrak{a}}\right) \\
& +\sum_{N_{\mathfrak{a}} \leqq x}\left\{-\mu(\mathfrak{a}) \alpha(\mathfrak{a}) \log N \mathfrak{a} C\left(\phi \alpha, \frac{x}{N \mathfrak{a}}\right)\right\} .
\end{aligned}
$$


By (1.5) and (1.7) the first term is just

$$
\begin{aligned}
\log x \sum_{N \mathfrak{a} \leqq x} \alpha^{-1}(\mathfrak{a}) & C\left(\phi \alpha, \frac{x}{N \mathfrak{a}}\right) \\
& =\log x C\left(\alpha^{-1} \phi \alpha, x\right)=\log x C(\phi, x) .
\end{aligned}
$$

Next we note that $-\mu(\mathfrak{a}) \alpha(\mathfrak{a}) \log N \mathfrak{a}=\left(\alpha^{-1}\right)^{\prime}(\mathfrak{a})$ and hence by (1.7), the second term is just

$$
\begin{aligned}
\sum_{N \mathfrak{a} \leqq x}\left(\alpha^{-1}\right)^{\prime}(\mathfrak{a}) & C\left(\phi \alpha, \frac{x}{N \mathfrak{a}}\right) \\
= & C\left(\left(-\alpha^{-1}\right)^{\prime} \phi \alpha, x\right)=C\left(-\left|\frac{\alpha^{\prime}}{\alpha^{2}}\right| \phi \alpha, x\right)=C\left(-\left|\frac{\alpha^{\prime}}{\alpha}\right| \phi, x\right) \\
= & \sum_{N_{\mathfrak{a}} \leqq x} \alpha(\mathfrak{a}) \Lambda(\mathfrak{a}) C\left(\phi, \frac{x}{N \mathfrak{a}}\right)
\end{aligned}
$$

by (1.3), (1.6), and (1.7). This proves the theorem.

Before we proceed we need a few preliminary estimates. First of all:

(1.9) There exists $\lambda>0$ such that $C(\zeta, x)=\Sigma_{N_{\mathfrak{a} \leqq x}} 1=\lambda x+O\left(x^{1-1 / n}\right)$ where $n$ is the degree of the field $k$ over the rationals, this result can be found in [2].

Suppose $\alpha \in D, \alpha(\mathfrak{a}) \geqq 0$ for all $\mathfrak{a} \in I$ and there exists $\xi>0$ such that $C(\alpha, x)=\xi x+O\left(x^{1-1 / n}\right) ;$ then:

$$
\sum_{y<N \mathfrak{a} \leqq x} \frac{1}{N \mathfrak{a}^{1-\delta}}=O\left(x^{\delta}-y^{\delta}\right), \quad 0<\delta<1, \quad x \rightarrow \infty .
$$

(1.11) There exists $\beta$ such that

$$
\begin{aligned}
& \sum_{N \mathfrak{a} \leqq x} \frac{\alpha(\mathfrak{a})}{N \mathfrak{a}}=\xi\{\log x+\beta\}+O\left(x^{-1 / n}\right), \quad x \rightarrow \infty . \\
& \sum_{N \mathfrak{a} \leqq x} \alpha(\mathfrak{a}) \log N \mathfrak{a}=\xi\{x \log x-x\}+O\left(x^{1-1 / n} \log x\right), \quad x \rightarrow \infty . \\
& \sum_{N \mathfrak{a} \leqq x} \alpha^{2}(\mathfrak{a})=C\left(\alpha^{2}, x\right)=\xi^{2}[x \log x+(2 \beta-1) x]+O\left(x^{1-1 / 2 n}\right), x \rightarrow \infty . \\
& \sum_{N \mathfrak{a} \leqq x} \alpha(\mathfrak{a}) \frac{\log \frac{x}{N \mathfrak{a}}}{N \mathfrak{a}^{1-\delta}}=O\left(x^{\delta}\right), \quad 0<\delta<1, \quad x \rightarrow \infty . \\
& \sum_{N_{\mathfrak{a}} \leqq x} \alpha(\mathfrak{a}) \frac{\log N \mathfrak{a}}{N \mathfrak{a}}=\xi \frac{\log ^{2} x}{2}+O(1), \quad x \rightarrow \infty .
\end{aligned}
$$

The proofs of (1.10)-(1.15) are based on summation by parts and use of $C(\alpha, x)=\xi x+O\left(x^{1-1 / n}\right)$.

Four well-known results of elementary number theory will now be derived by use of the estimates and identities listed above.

THEOREM 1.2. $\Sigma_{N_{\mathfrak{a} \leqq x}}(\mu(\mathfrak{a}) / N \mathfrak{a})=O(1)$. 
Proof.

$$
\begin{aligned}
x \sum_{N \mathfrak{a} \leqq x} \frac{\mu(\mathfrak{a})}{N \mathfrak{a}} & =\sum_{N \mathfrak{a} \leqq x} \mu(\mathfrak{a}) \frac{x}{N \mathfrak{a}} \\
& =\sum_{N \mathfrak{a} \leqq x} \mu(\mathfrak{a}) \frac{1}{\lambda}\left\{C\left(\zeta,-\frac{x}{N \mathfrak{a}}\right)+O\left(\left(\frac{x}{N \mathfrak{a}}\right)^{1-1 / n}\right)\right\} \\
& =\frac{1}{\lambda}+O\left(x^{1-1 / n}\right) \sum_{N \mathfrak{a} \leqq x} \frac{1}{N \mathfrak{a}^{1-1 / n}}=O(x) .
\end{aligned}
$$

THEOREM 1.3. $\quad \sum_{N_{\mathfrak{a}} \leqq x}(\mu(\mathfrak{a}) / N(\mathfrak{a})) \log (x / N \mathfrak{a})=O(1)$.

\section{Proof.}

$$
\begin{aligned}
\sum_{N \mathfrak{a} \leqq x} \frac{\mu(\mathfrak{a})}{N \mathfrak{a}} \log \frac{x}{N \mathfrak{a}}= & \sum \frac{\mu(\mathfrak{a})}{N \mathfrak{a}}\left\{\frac{1}{\lambda} \sum_{N \mathfrak{b} \leqq x / N \mathfrak{a}} \frac{1}{N \mathfrak{b}}-\beta+O\left(\left(\frac{x}{N \mathfrak{a}}\right)^{-1 / n}\right)\right\} \\
= & \frac{1}{\lambda} C\left(\alpha \alpha^{-1}, x\right)-\beta \sum_{N \mathfrak{a} \leqq x} \frac{\mu(\mathfrak{a})}{N \mathfrak{a}} \\
& +O\left(x^{1-n}\right) \sum_{N \mathfrak{a} \leqq x} \frac{1}{N \mathfrak{a}^{1-1 / n}} \quad \text { where } \alpha(\mathfrak{a})=\frac{1}{N \mathfrak{a}} .
\end{aligned}
$$

The result then follows from Theorem 1.2 and (1.10).

THEOREM 1.4. $\psi(x)=\sum_{N_{\mathfrak{a}} \leqq} \Lambda(\mathfrak{a})=O(x)$.

Proof.

$$
\begin{aligned}
\sum_{N \mathfrak{a} \leqq x} \Lambda(\mathfrak{a})= & C\left(-\zeta^{\prime} / \zeta, x\right) \\
= & \sum_{N \mathfrak{a} \leqq x} \mu(\mathfrak{a}) \sum_{N \mathfrak{b} \leqq x / N \mathfrak{a}} \log N \mathfrak{b} \\
= & \sum_{N \mathfrak{a} \leqq x} \mu(\mathfrak{a})\left[\lambda \frac{x}{N \mathfrak{a}} \log \frac{x}{N \mathfrak{a}}-\frac{\lambda x}{N \mathfrak{a}}+O\left(\left(\frac{x}{N \mathfrak{a}}\right)^{1-1 / n} \log \frac{x}{N \mathfrak{a}}\right)\right] \\
= & \lambda x\left[\sum_{N \mathfrak{a} \leqq x} \frac{\mu(\mathfrak{a})}{N \mathfrak{a}} \log \frac{x}{N \mathfrak{a}}-\sum_{N \mathfrak{a} \leqq x} \frac{\mu(\mathfrak{a})}{N \mathfrak{a}}\right] \\
& +O\left(x^{1-1 / n}\right) \sum_{N \mathfrak{a} \leqq x} \frac{\log \frac{1}{N \mathfrak{a}}}{N \mathfrak{a}^{1-1 / n}}=O(x)
\end{aligned}
$$

by Theorems 1.2 and 1.3 and by (1.14).

THEOREM 1.5. $\Sigma_{N_{\mathfrak{a}} \leqq x}(\Lambda(\mathfrak{a}) / N \mathfrak{a})=\log x+O(1)$.

Proof.

$$
\begin{aligned}
x \sum_{N \mathfrak{a} \leqq x} \frac{\Lambda(\mathfrak{a})}{N \mathfrak{a}} & =\sum_{N \mathfrak{a} \leqq x} \Lambda(\mathfrak{a})\left\{\frac{1}{\lambda} C\left(\zeta, \frac{x}{N \mathfrak{a}}\right)+O\left(\left(\frac{x}{N \mathfrak{a}}\right)^{1-1 / n}\right)\right\} \\
& =\frac{1}{\lambda} C\left(-\frac{\zeta^{\prime}}{\zeta} \zeta, x\right)+O\left(x^{1-1 / n}\right) \sum_{N \mathfrak{a} \leqq x} \frac{\Lambda(\mathfrak{a})}{N \mathfrak{a}^{1-1 / n}} .
\end{aligned}
$$


Summing by parts and using the fact that $\Sigma_{N_{\mathfrak{a}} \leqq} \Lambda(\mathfrak{a})=O(x)$ we see that the second term is $O(x)$. The first term is just

$$
\frac{1}{\lambda} C\left(-\zeta^{\prime}, x\right)=\frac{1}{\lambda} \sum_{N \mathfrak{a} \leqq x} \log N a=x \log x+O(x)
$$

by (1.12). This proves the result.

We return to (1.8). This identity says that if we want information about $C(\phi, x)$ for some $\phi \in D$, an estimate for $C(\phi \alpha, x)$ for some strongly multiplicative $\alpha \in D$ might help. If we let $\phi=-\zeta^{\prime} / \zeta-(1 / \lambda) \zeta+2 \beta e$ then $C(\phi, x)=\Sigma_{N_{\mathfrak{a}} \leqq x} \Lambda(\mathfrak{a})-x+o(x)$ and hence the proposition $C(\phi, x)=o(x)$ is the prime ideal theorem. If we let $\alpha=\zeta$, then

$$
C(\phi \alpha, x)=C\left(-\zeta^{\prime}-\frac{1}{\lambda} \zeta^{2}+2 \beta \zeta, x\right)=O\left(x^{1-1 / 2 n}\right)
$$

by (1.12), (1.13), and (1.9) and hence

$$
\sum_{N \mathfrak{a} \leqq x} \mu(\mathfrak{a}) \alpha(\mathfrak{a}) \log \frac{x}{N \mathfrak{a}} C\left(\phi \alpha, \frac{x}{N \mathfrak{a}}\right)=\sum_{N \mathfrak{a} \leqq x} O\left(\left(\frac{x}{N \mathfrak{a}}\right)^{1-\varepsilon}\right)
$$

for sufficiently small $\varepsilon>0$ and this is just $O(x)$ by (1.10). Therefore by Theorem 1.1

or,

$$
C(\phi, x) \log x+\sum_{N \mathfrak{a} \leqq x} \Lambda(\mathfrak{a}) C\left(\phi, \frac{x}{N \mathfrak{a}}\right)=O(x)
$$

$$
\begin{aligned}
& \psi(x) \log x+\sum_{N \mathfrak{a} \leqq x} \Lambda(\mathfrak{a}) \psi\left(\frac{x}{N \mathfrak{a}}\right) \\
& =\log x C\left(\frac{1}{\lambda^{\prime}}, x\right)+\sum_{N \mathfrak{a} \leqq x} \Lambda(\mathfrak{a}) C\left(\frac{1}{\lambda} \zeta, \frac{x}{N \mathfrak{a}}\right)+O(x) \\
& =x \log x+\frac{1}{\lambda} C\left(-\zeta^{\prime}, x\right)+O(x)=2 x \log x+O(x)
\end{aligned}
$$

This is just Selberg's fundamental formula for algebraic number fields.

However this technique will yield a stronger result. If we let $\alpha \in D$ be defined by $\alpha(\mathfrak{a})=1 / N \mathfrak{a}$ and $\phi=-\alpha^{\prime} / \alpha-(1 / \lambda) \alpha+2 \beta e$, then

$$
C(\phi, x)=\sum_{N \mathfrak{a} \leqq x} \frac{\Lambda(\mathfrak{a})}{N \mathfrak{a}}-\log x+\beta+o(1) .
$$

Since it is known that $\Sigma_{N \mathfrak{a} \leqq x}(\Lambda(\mathfrak{a}) / N \mathfrak{a})=\log x-\beta+o(1)$ is an elementary equivalent of the prime ideal theorem, so is the condition $C(\phi, x)=o(1)$. Using the estimates already derived it is not difficult to show that

$$
C(\phi \alpha, x)=C\left(-\alpha^{\prime}-\frac{1}{\lambda} \alpha^{2}+2 \beta \alpha, x\right)=A+O\left(x^{-1 / 2 n}\right)
$$


for some constant $A$. Therefore

$$
\begin{aligned}
& \sum_{N \mathfrak{a} \leqq x} \mu(\mathfrak{a}) \alpha(\mathfrak{a}) \log \frac{x}{N \mathfrak{a}} C\left(\phi \alpha, \frac{x}{N \mathfrak{a}}\right) \\
& \quad=A \sum_{N \mathfrak{a} \leqq x} \frac{\mu(\mathfrak{a})}{N \mathfrak{a}} \log \frac{x}{N \mathfrak{a}}+O\left(x^{-1 / 2 n}\right) \sum_{N \mathfrak{a} \leqq x} \frac{\log (x / N \mathfrak{a})}{N \mathfrak{a}^{1-1 / 2 \mathfrak{n}}}=O(1)
\end{aligned}
$$

by Theorem 1.3 and (1.14).

Hence we have

$$
\log x C(\phi, x)+\sum_{N \mathfrak{a} \leqq x} \frac{\Lambda(\mathfrak{a})}{N \mathfrak{a}} C\left(\phi, \frac{x}{N \mathfrak{a}}\right)=O(1) .
$$

Using the definition of $\phi$ we get

$$
\begin{aligned}
\log x \sum_{N \mathfrak{a} \leqq x} & \frac{\Lambda(\mathfrak{a})}{N \mathfrak{a}}+\sum_{N \mathfrak{a} \mathfrak{x}} \frac{\Lambda(\mathfrak{a}) \Lambda(\mathfrak{b})}{N \mathfrak{a} \mathfrak{b}} \\
= & \frac{1}{\lambda} \log x C(\alpha, x)-2 \beta \log x+\frac{1}{\lambda} \sum_{N \mathfrak{a} \leqq x} \frac{\Lambda(\mathfrak{a})}{N \mathfrak{a}} C\left(\alpha, \frac{x}{N \mathfrak{a}}\right) \\
& -2 \beta \sum_{N \mathfrak{a} \leqq x} \frac{\Lambda(\mathfrak{a})}{N \mathfrak{a}}+O(1) .
\end{aligned}
$$

We note that the third term is just $\lambda^{-1} C\left(-\left(\alpha^{\prime} / \alpha\right) \cdot \alpha, x\right)=\lambda^{-1} \sum_{N \mathfrak{a} \leqq x}(\log N \mathfrak{a}) / N \mathfrak{a}$. Hence every term can be estimated up to a constant and the end result is:

(1.16) $\log x \sum_{N \mathfrak{a} \leqq x} \frac{\Lambda(\mathfrak{a})}{N \mathfrak{a}}+\sum_{N \mathfrak{a} \mathfrak{b} \leqq x} \frac{\Lambda(\mathfrak{a}) \Lambda(\mathfrak{b})}{N \mathfrak{a} \mathfrak{b}}=\frac{3}{2} \log ^{2} x-3 \beta \log x+O(1)$.

A simple elementary argument shows that

$$
\begin{aligned}
\log x \sum_{N \mathfrak{a} \leqq x} \frac{\Lambda(\mathfrak{a})}{N(\mathfrak{a})}-\sum_{N_{\mathfrak{a} \leqq x}} \frac{\Lambda(\mathfrak{a}) \log N \mathfrak{a}}{N \mathfrak{a}} & =\sum_{N \mathfrak{a} \leqq x} \frac{\Lambda(\mathfrak{a})}{N \mathfrak{a}} \log \frac{x}{N \mathfrak{a}} \\
& =\frac{\log ^{2} x}{2}+\beta \log x+O(1)
\end{aligned}
$$

and hence it follows from (1.16) that

$$
\sum_{N \mathfrak{a} \leqq x} \frac{\Lambda(\mathfrak{a}) \log N \mathfrak{a}}{N \mathfrak{a}}+\sum_{N \mathfrak{a} \mathfrak{b} \leqq x} \frac{\Lambda(\mathfrak{a}) \Lambda(\mathfrak{b})}{N \mathfrak{a} \mathfrak{b}}=\log ^{2} x-2 \beta \log x+O(1) .
$$

This result was first proved (by "elementary" methods) by Amitsur [1]. His proof is somewhat more complicated as it involves the use of his "symbolic calculus." It is easy to show that (1.17) implies Selberg's fundamentalformula (Amitsur [1]). 
Before we proceed we need a few more definitions.

Definition 1.3. Let $\mathrm{m}$ be an integral ideal of $k$, then $A^{(\mathfrak{m})}$ is the group of fractional ideals of $k$ prime to $\mathfrak{m}$ and $H^{(\mathfrak{m})}$ is the group of principal ideals $(\alpha)$ where

(a) $\alpha$ is totally positive,

(b) $v_{\mathfrak{p}}(\alpha-1) \geqq v_{\mathfrak{p}}(\mathfrak{m})$ for all $\mathfrak{p} \geqq \mathfrak{m}$, i.e., for all prime ideals $\mathfrak{p}$ dividing $\mathfrak{m}$ the exponent of $\mathfrak{p}$ in the unique factorization of $(\alpha-1)$ is greater than the exponent of $p$ in the unique factorization of $m$.

We assume the following facts found in Landau [1].

(1.18) $A^{(\mathfrak{m})} / H^{(\mathfrak{m})}$ is a finite group of order $h$.

(1.19) If $g \in G_{\mathfrak{m}}=A^{(\mathfrak{m})} / H^{(\mathfrak{m})}$ then $\Sigma_{N_{\mathfrak{a}} \leqq x, \mathfrak{a} \in g} 1=\tau x+O\left(x^{1-1 / n}\right)$ where $\tau>0$ is independent of $g$ and $n$ is the degree of $k$ over the rationals. If $\chi$ is a character of $G_{\mathfrak{m}}$ then for any $\mathfrak{a} \in I$ we define $\chi(\mathfrak{a})=\chi\left(\mathfrak{a} H^{(\mathfrak{m})}\right)$ if $\mathfrak{a} \in A^{(\mathfrak{m})}$, and $\chi(\mathfrak{a})=0$ otherwise. Then $\chi$ defines a strongly multiplicative formal Dirichlet series. From (1.19) and elementary properties of characters of finite abelian groups one obtains

(1.20) For any nonprincipal character $\chi$ of $G_{\mathfrak{m}}$ (i.e., $\left.\chi \neq \equiv 1\right)$ we have

$$
\sum_{N \mathfrak{a} \leqq x} \chi(\mathfrak{a})=O\left(x^{1-1 / n}\right)
$$

(1.21) If $\varepsilon$ is the principal character of $G_{\mathfrak{m}}$ (i.e., $\varepsilon \equiv 1$ ) then

$$
\sum_{N_{\mathfrak{a}} \leqq x} \varepsilon(\mathfrak{a})=\sum_{N_{\mathfrak{a}} \leqq x, \mathfrak{a} \in A} 1=\omega x+O\left(x^{1-1 / \mathfrak{m})}\right),
$$

where $\omega=h \tau>0$.

If $\varepsilon$ is the principal character of $G_{\mathfrak{m}}$ then

$$
\log x \sum_{N \mathfrak{a} \leqq x} \varepsilon(\mathfrak{a}) \Lambda(\mathfrak{a})+\sum_{N \mathfrak{a} \mathfrak{b} \leqq x} \varepsilon(\mathfrak{a} \mathfrak{b}) \Lambda(\mathfrak{a}) \Lambda(\mathfrak{b})=2 x \log x+O(x) .
$$

This follows when one notices that the difference between the left-hand side of (1.22) and the left-hand side of Selberg's fundamental formula is $O(x)$.

If $\chi$ is a nonprincipal character of $G_{\mathfrak{m}}$ then we apply Theorem 1.1 with $\phi=-\chi^{\prime} / \chi$ and $\alpha=\chi$. Now

$$
C(\phi \alpha, x)=C\left(-\chi^{\prime}, x\right)=\sum_{N_{\mathfrak{a}} \leqq x} \chi(\mathfrak{a}) \log N \mathfrak{a}=O\left(x^{1-\varepsilon}\right)
$$

for $0<\varepsilon<1 / n$, as can be seen by summation by parts and application of (1.20). Therefore,

$$
\sum_{N \mathfrak{a} \leqq x} \mu(\mathfrak{a}) \alpha(\mathfrak{a}) \log \frac{x}{N \mathfrak{a}} C\left(\phi \alpha, \frac{x}{N \mathfrak{a}}\right)=\sum_{N \mathfrak{a} \leqq x} O\left(\left(\frac{x}{N \mathfrak{a}}\right)^{1-\varepsilon / 2}\right)=O(x)
$$

as we have seen. It follows that

$$
\log x \sum_{N_{\mathfrak{a}} \leqq x} \chi(\mathfrak{a}) \Lambda(\mathfrak{a})+\sum_{N_{\mathfrak{a} \mathfrak{b} \leqq x}} \chi(\mathfrak{a} \mathfrak{b}) \Lambda(\mathfrak{a}) \Lambda(\mathfrak{b})=\eta_{\chi}(x),
$$


where $\eta_{\chi}(x)=O(x)$ for $\chi \neq \varepsilon$ and $\eta_{\chi}(x)=2 x \log x+O(x)$ for $\chi=\varepsilon$. Rearranging, we have

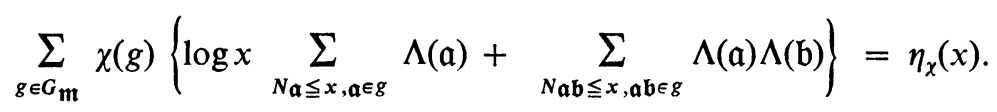

Solving these equations we get, for each $g \in G$

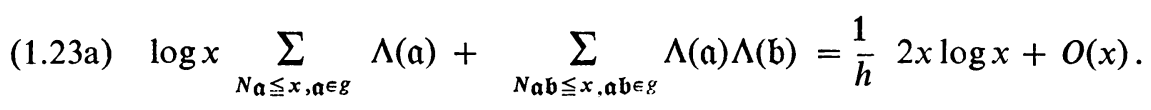

This is Selberg's fundamental formula for ideals in ideal classes modulo $m$.

Now we show how (1.17) can be generalized to ideal classes. From (1.11) there exists $c_{0}$ such that

$$
\sum_{N \mathfrak{a} \leqq x} \frac{\varepsilon(\mathfrak{a})}{N \mathfrak{a}}=\omega\left[\log x+c_{0}\right]+O\left(x^{-1 / n}\right) .
$$

Furthermore we can show that

$$
\begin{aligned}
& \sum_{N \mathfrak{a} \leqq x} \frac{\varepsilon(\mathfrak{a}) \mu(\mathfrak{a})}{N \mathfrak{a}}=O(1), \\
& \sum_{N \mathfrak{a} \leqq x} \frac{\varepsilon(\mathfrak{a}) \mu(\mathfrak{a})}{N \mathfrak{a}} \log \frac{x}{N \mathfrak{a}}=O(1), \\
& \sum_{N \mathfrak{a} \leqq x} \frac{\varepsilon(\mathfrak{a}) \Lambda(\mathfrak{a})}{N \mathfrak{a}}=\log x+O(1) .
\end{aligned}
$$

The proofs of (1.24)-(1.26) are almost identical with the proofs of (1.2), (1.3), and (1.5). The important thing is that $\varepsilon$ defines a strongly multiplicative formal Dirichlet series such that $C(\varepsilon, x)=\omega x+O\left(x^{1-1 / n}\right)$ for some $\omega>0$. With these results we can prove

$$
\begin{array}{r}
\log x \sum_{N \mathfrak{a} \leqq x} \frac{\varepsilon(\mathfrak{a}) \Lambda(\mathfrak{a})}{N \mathfrak{a}}+\sum_{N \mathfrak{a} \mathfrak{x} \frac{\varepsilon}{\varepsilon(\mathfrak{a} \mathfrak{b}) \Lambda(\mathfrak{a}) \Lambda(\mathfrak{b})}}^{N a \mathfrak{b}} \\
=\frac{3}{2} \log ^{2} x-3 c_{0} \log x+O(1)
\end{array}
$$

in exactly the same way that (1.16) was proved. Furthermore from (1.27) we can deduce

$$
\begin{aligned}
\sum_{N \mathfrak{a} \leqq x} \frac{\varepsilon(\mathfrak{a}) \Lambda(\mathfrak{a}) \log N \mathfrak{a}}{N \mathfrak{a}}+\sum_{N_{\mathfrak{a} \mathfrak{b} \leqq x}} \frac{\varepsilon(\mathfrak{a} \mathfrak{b}) \Lambda(\mathfrak{a}) \Lambda(\mathfrak{b})}{N \mathfrak{a} \mathfrak{b}} \\
=\log ^{2} x-2 c_{0} \log x+O(1)
\end{aligned}
$$

in the same way that (1.17) was deduced from (1.16).

In order to get the corresponding results for the nonprincipal characterswe proceed along different lines. Moreover we need a result that can be proved by elementary methods (Shapiro and Forman [1]). 
(1.29) If $\chi$ is a nonprincipal character of $G_{\mathfrak{m}}$ then

$$
\sum_{N \mathfrak{a} \leq x} \frac{\chi(\mathfrak{a}) \mu(\mathfrak{a})}{N \mathfrak{a}}=O(1) .
$$

We consider the identity $\left(\alpha^{\prime} / \alpha\right)^{\prime}+\left(\alpha^{\prime} / \alpha\right)=\alpha^{\prime \prime} / \alpha$ and let $\alpha$ be defined by $\alpha(\mathfrak{a})=\chi(\mathfrak{a}) / N \mathfrak{a}$. Note that

$$
C\left(\left(\frac{\alpha^{\prime}}{\alpha}\right)^{\prime}, x\right)=\sum_{N \mathfrak{a} \leqq x} \frac{\chi(\mathfrak{a}) \Lambda(\mathfrak{a}) \log N \mathfrak{a}}{N \mathfrak{a}}
$$

and

$$
C\left(\left(\frac{\alpha^{\prime}}{\alpha}\right)^{2}, x\right)=\sum_{N \mathfrak{a} \leqq x} \frac{\chi(\mathfrak{a} \mathfrak{b}) \Lambda(\mathfrak{a}) \Lambda(\mathfrak{b})}{N \mathfrak{a} \mathfrak{b}} .
$$

By summation by parts we see that

$$
C\left(\alpha^{\prime \prime}, x\right)=\sum_{N \mathfrak{a} \leqq x} \frac{\chi(\mathfrak{a})}{N \mathfrak{a}} \log ^{2} N \mathfrak{a}=b_{0}+O\left(x^{-\varepsilon}\right)
$$

for some constant $b_{0}$ and sufficiently small $\varepsilon>0$. Hence

$$
\begin{aligned}
C\left(\frac{\alpha^{\prime \prime}}{\alpha}, x\right) & =\sum_{N_{\mathfrak{a}} \leqq x} \frac{\chi(\mathfrak{a}) \mu(\mathfrak{a})}{N \mathfrak{a}}\left\{b_{0}+O\left(\left(\frac{x}{N \mathfrak{a}}\right)^{-\mathfrak{e}}\right)\right\} \\
& =b_{0} \sum_{N \mathfrak{a} \leqq x} \frac{\chi(\mathfrak{a}) \mu(\mathfrak{a})}{N \mathfrak{a}}+O\left(x^{-\mathfrak{\varepsilon}}\right) \sum_{N \mathfrak{a} \leq x} N \mathfrak{a}^{\varepsilon-1}=O(1) .
\end{aligned}
$$

It follows that

$$
\sum_{N \mathfrak{a} \leqq x} \frac{\chi(\mathfrak{a}) \Lambda(\mathfrak{a}) \log N \mathfrak{a}}{N \mathfrak{a}}+\sum_{N_{\mathfrak{a} \mathfrak{b} \leqq x}} \frac{\chi(\mathfrak{a} \mathfrak{b}) \Lambda(\mathfrak{a}) \Lambda(\mathfrak{b})}{N \mathfrak{a} \mathfrak{b}}=\eta_{\chi}(x)
$$

where $\eta_{\chi}(x)=\log ^{2} x-2 c_{0} \log x+O(1)$ if $\chi=\varepsilon$ and $\eta_{x}(x)=O(1)$ if $\chi \neq \varepsilon$. We can rewrite this as

$$
\sum_{g \in G_{\mathfrak{m}}} \chi(g)\left\{\sum_{N_{\mathfrak{a}} \leqq x, \mathfrak{a} \in g} \frac{\Lambda(\mathfrak{a}) \log N \mathfrak{a}}{N \mathfrak{a}}+\sum_{N_{\mathfrak{a} b} \leq x, \mathfrak{b} \in \mathfrak{g}} \frac{\Lambda(\mathfrak{a}) \Lambda(\mathfrak{b})}{N \mathfrak{a} \mathfrak{b}}\right\}=\eta_{\chi}(x)
$$

and solving we get, for each $g \in G_{\mathfrak{m}}$

$$
\sum_{N_{\mathfrak{a}} \leq x, \mathfrak{a} \in g} \frac{\Lambda(\mathfrak{a}) \log N \mathfrak{a}}{N \mathfrak{a}}+\sum_{N_{\mathfrak{a} b} \leq x, \mathfrak{a} \mathfrak{b} \in \mathfrak{g}} \frac{\Lambda(\mathfrak{a}) \Lambda(\mathfrak{b})}{N \mathfrak{a} \mathfrak{b}}=\frac{1}{h}\left\{\log ^{2} x-2 c_{0} \log x\right\}+O(1) .
$$

II. Tauberian methods. Now let $m$ be an integral ideal of $k$ and let $\chi$ be a nonprincipal character of $G_{\mathfrak{m}}$. Then by (1.23) we have:

$$
f(x)+\frac{1}{\log x} \sum_{n \leqq x} \alpha(n) f\left(\frac{x}{n}\right) \rightarrow 0 \text { as } x \rightarrow \infty,
$$


where

and

$$
f(x) \frac{1}{x}=\sum_{N \mathfrak{a} \leqq x} \chi(\mathfrak{a}) \Lambda(\mathfrak{a}),
$$

$$
\alpha(n)=\sum_{N \mathfrak{a}=n} \frac{\chi(\mathfrak{a}) \Lambda(\mathfrak{a})}{N \mathfrak{a}} .
$$

Furthermore if $\varepsilon$ is the principal character of $G_{\mathfrak{m}}$ then by (1.23), formula (2.2) also holds for

$$
f(x)=\frac{1}{x}\left[\sum_{N \mathfrak{a} \leqq x} \varepsilon(\mathfrak{a}) \Lambda(\mathfrak{a})-x\right],
$$

and

$$
\alpha(n)=\sum_{N_{\mathfrak{a}} \leqq x} \frac{\varepsilon(\mathfrak{a}) \Lambda(\mathfrak{a})}{N \mathfrak{a}} .
$$

We would like a result stating that with appropriate hypotheses on $f$ and $\alpha$, the condition (2.1) implies $f(x)=o(1)$ as $x \rightarrow \infty$. The condition

$$
\sum_{N_{\mathfrak{a}} \leqq x} \chi(\mathfrak{a}) \Lambda(\mathfrak{a})=o(x) \text { for } \chi \neq \varepsilon \text { and } x+o(x) \text { for } \chi=\varepsilon,
$$

is a simple equivalent of the theorem on equidistribution of prime ideals in ideal classes.

In his paper, Some results on arithmetic functions, Amitsur [2] states the following:

Let $g(n)$ be a non-negative function such that

$$
\sum_{n \leqq x} g(n)=a \log ^{m} x+b \log ^{m-1} x+o\left(\log ^{m-1} x\right), \quad m \geqq 1, a>0 .
$$

"Let $f(x)$ be a complex-valued function such that

$$
\begin{gathered}
f(x)=O(1), \\
\sum_{n \leqq x} \frac{f(n)}{n}=O(1), \\
f(t x)-f(x)=o(1) \text { as }(t, x) \rightarrow(1, \infty) .
\end{gathered}
$$

"Then the condition

$$
|f(x)| \log ^{m} x \leqq \frac{1}{a} \sum_{n \leqq x} g(n)\left|f\left(\frac{x}{n}\right)\right|+o\left(\log ^{m} x\right)
$$

implies that $f(x)=o(1)$."

By (2.1) we have

$$
|f(x)| \log x \leqq \sum_{n \leqq x} g(n)\left|f\left(\frac{x}{n}\right)\right|+o(\log x),
$$

where $f$ is given either by (2.2) or (2.4) and $g(n)=\Sigma_{N_{\mathfrak{a}}=n}(\Lambda(\mathfrak{a}) / N \mathfrak{a})$. 
(A. 1) follows from the prime ideal theorem, (A.5) is just (2.7) and (A. 2)-(A.4) can be verified by elementary methods. However, the proposition is not true as it stands. In fact, one easily checks that the functions $f(x)=e^{i \log x}$ and $g(n)=1 / n$, provide a counterexample.

It should be pointed out that if we have (1.23) for some nonprincipal character $\chi$ of $G_{\mathfrak{m}}$, and we know (1.23a) for all $h \in G_{\mathfrak{m}}$ then it is possible to give a proof very similar to the one in Selberg [2]. However we will take a slightly more general approach.

Suppose $f$ is a complex-valued function defined on $(0, \infty)$ and $\alpha$ is a non-negative function defined on integral ideals; suppose we have:

$$
f(x)+\frac{1}{\log x} \sum_{N \mathfrak{a} \leqq x} \chi(\mathfrak{a}) \alpha(\mathfrak{a}) f\left(\frac{x}{N} \overline{\mathfrak{a}}\right) \rightarrow 0 .
$$

For $h \in G_{\mathfrak{m}}$ we multiply by $\chi(h)$ and rewrite as follows:

$$
\chi(h) f(x)+\sum_{g \in \mathbf{G}_{\mathfrak{m}}} \frac{1}{\log x} \sum_{N_{\mathfrak{a}} \leqq x, \mathfrak{a} \in g} \alpha(\mathfrak{a}) \chi(h g) f\left(\frac{x}{N \mathfrak{a}}\right) \rightarrow 0 .
$$

Or, with $f_{h}(x)=\chi(h) f(x)$, we have:

$$
f_{h}(x)+\sum_{g \in G_{\mathfrak{m}}} \frac{1}{\log x} \sum_{N \mathfrak{a} \leqq x, \mathfrak{a} \in g h^{-1}} \alpha(\mathfrak{a}) f_{g}\left(\frac{x}{N \mathfrak{a}}\right) \rightarrow 0
$$

for all $h \in G_{\mathfrak{m}}$. If the $f_{g}$ are replaced by either their real or imaginary parts the relation still holds. Finally, for each $h \in G_{\mathfrak{m}}$ we have:

$$
\left|f_{h}(x)\right| \leqq \sum_{g \in G_{\mathfrak{m}}} \frac{1}{\log x} \sum_{n \leqq x} \beta_{h, g}(n)\left|f_{g}\left(\frac{x}{n}\right)\right|+o(1),
$$

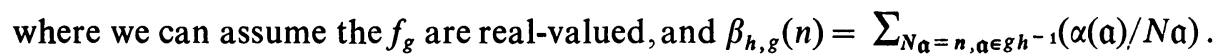
Starting with the single relation, (2.8), involving complex-valued functions we arrive at the system, (2.9), of relations involving real-valued functions and where the coefficients $\beta_{h, g}$ are even non-negative. It is from this point of view that we approach the problem.

It is very convenient, although not necessary, to discuss the Tauberian theorem in terms of Lebesgue-Stieltjes integrals rather than finite sums.

Let $k(x)$ be monotone increasing, defined on $(0, \infty)$, we assume that $k$ is normalized to be continuous from the left at each point. For any interval $[a, b)$ we define its measure to be $k(b)-k(a)$, this measure has a unique extension to the Borel sets. If $f(x)$ is integrable for this measure we denote its integral by $\int_{0}^{\infty} f(x) d k(x)$. We will also need the following results which can be found in Pitt [1], [2]. 
(2.10) Suppose $h, k$ are monotone increasing on $(0, \infty)$ then

$$
\int_{0}^{x} h(x-y) d k(y)=\int_{0}^{x} k(x-y) d h(y) .
$$

(2.11) If $k(x)$ is monotone increasing and absolutely continuous and $f$ is integrable with respect to $k$, then $\int_{0}^{\infty} f(x) d k(x)=\int_{0}^{\infty} f(x) k^{\prime}(x) d x$.

(2.12) Suppose $h, k$ are monotone increasing and $f$ is Borel measurable and bounded in any finite interval then

$$
\int_{0}^{x}\left\{\int_{0}^{x-y} f(x-y-t) d h(t)\right\} d k(y)=\int_{0}^{x} f(x-y) d l(y),
$$

where

$$
l(y)=\int_{0}^{y} h(y-t) d k(t) .
$$

From now on we assume that all functions mentioned are Borel measurable and defined on $(0, \infty)$, and all measures $k(x)$ are continuous from the left.

Lemma 2.1. Suppose $f(x)$ is real-valued and bounded. Suppose $k(x)$ is monotone, $k(0)=0$, and $k$ is non-negative and $k(x) \sim a F(x)$ for some function $F(x)$, and $k(x)-k\left(x-x_{0}\right)=o(F(x))$ for each fixed $x_{0}$. Let $S=\limsup _{x \rightarrow \infty} f(x)$, $s=\liminf _{x \rightarrow \infty} f(x)$ then:

$$
\begin{aligned}
a s & \leqq \liminf _{x \rightarrow \infty} \frac{1}{F(x)} \int_{0}^{x} f(x-y) d k(y) \\
& \leqq \limsup _{x \rightarrow \infty} \frac{1}{F(x)} \int_{0}^{x} f(x-y) d k(y) \leqq a S .
\end{aligned}
$$

Proof. The middle inequality is clear. We prove only that

$$
\limsup _{x \rightarrow \infty} \frac{1}{F(x)} \int_{0}^{x} f(x-y) d k(y) \leqq a S
$$

since the other inequality is similar. Let $\varepsilon>0$ be given, then choose $x_{0}$ such that $f(t)<S+\varepsilon$ for all $t \geqq x_{0}$. We split the integral as follows:

$$
\frac{1}{F(x)} \int_{0}^{x} f(x-y) d k(y)=\frac{1}{F(x)} \int_{0}^{x-x_{0}} f(x-y) d k(y)+\frac{1}{F(x)} \int_{x-x_{0}}^{x} f(x-y) d k(y) .
$$

The second term goes to zero, since it is bounded by $\left(k(x)-k\left(x-x_{0}\right)\right) M / F(x)$ where $M$ is a bound for $f$. Noting that $x-y \geqq x_{0}$ when $y \leqq x-x_{0}$ we see that the first term is bounded by

$$
(S+\varepsilon) \frac{k\left(x-x_{0}\right)}{F(x)}=(S+\varepsilon)\left\{\frac{k(x)}{F(x)}-\frac{k(x)-k\left(x-x_{0}\right)}{F(x)}\right\}
$$


which has the limit $a(S+\varepsilon)$ as $x \rightarrow \infty$. Since $\varepsilon$ was arbitrary the result follows.

The next lemma is essentially the same as a result of Selberg [1]. We include a proof since we use integrals instead of finite sums.

LEMMA 2.2. Suppose $f(x)$ is real-valued, that $f(x)-f(x-d) \rightarrow 0$ as $(x, d) \rightarrow(\infty, 0)$, and that $\int_{0}^{x} f(t) d t=O(1)$, then given $\varepsilon>0$ there exist $x_{0}, T$, and $d$ all greater than zero such that for all $x \geqq x_{0}$ the interval $(x, x+T)$ contains a subinterval of length $d$, throughout which $|f(t)|<\varepsilon$.

Proof. By hypothesis, there exists a constant $M$ such that $\left|\int_{x}^{x^{\prime}} f(t) d t\right| \leqq M$ for any $x, x^{\prime}$. We first choose $T$ so large that $M / T<\varepsilon / 2$. Next choose $x_{0}$ and $d$ so that $|f(x)-f(x-\delta)|<\varepsilon / 2$ for all $x \geqq x_{0}$ and $0<\delta<d$. We may also assume that $d<T / 2$. Consider the interval $(x, x+T)$ for some $x \geqq x_{0}$. If $f(t)$ does not change sign in this interval then $\inf _{t \in(x, x+T)}|f(t)| T \leqq\left|\int_{x}^{x+T} f(t) d t\right| \leqq M$ and hence $\inf _{t \in(x, x+T)}|f(t)| \leqq M / T<\varepsilon / 2$ and it follows that $|f(\tau)|<\varepsilon / 2$, for some $\tau \in(x, x+T)$. Now one of the intervals $(\tau, \tau+d)$ or $(\tau-d, \tau)$ is contained in $(x, x+T)$ since $d<T / 2$. If $t$ is in $(\tau, \tau+d)$ say, then $|f(t)|=|f(\tau+\delta)|$ $=|f(\tau)-(f(\tau)-f(\tau+\delta))| \leqq|f(\tau)|+|f(\tau)-f(\tau+\delta)|$ for some $0<\delta<d$. Hence $|f(t)|<\varepsilon$ in this interval. On the other hand if $f$ does change sign in this interval then there exists $\tau \in(x, x+T)$ such that $f(\tau)$ and $f(\tau+d)$ have opposite sign. In this case $|f(\tau)| \leqq|f(\tau)|+|f(\tau+d)|=|f(\tau)-f(\tau+d)|<\varepsilon / 2$. As before we can find an interval contained in $(x, x+T)$ with one end point at $\tau$ throughout which $|f(t)|<\varepsilon$.

From now on we assume that we have $N$ real-valued functions $f_{1}, \cdots, f_{N}$ defined on $(0, \infty)$ and $N^{2}$ monotone increasing non-negative functions $k_{i j}$, subject to the following conditions:

$$
\begin{gathered}
f_{i}(x)=O(1) ; i=1, \cdots, N . \\
\int_{0}^{x} f_{i}(t) d t=O(1) ; i=1, \cdots, N . \\
\left|f_{i}(x)\right| \leqq \sum_{i=1}^{N} \frac{1}{x} \int_{0}^{x}\left|f_{j}(x-y)\right| d k_{i j}(y)+o(1) ; i=1, \cdots, N . \\
f_{i}(x-d)-f_{i}(x) \rightarrow 0 \text { as }(x, d) \rightarrow(\infty, 0) . \\
k_{i j}(x) \sim \frac{x}{N} ; i, j=1, \cdots, N, k_{i j}(0+)=0 .
\end{gathered}
$$

(2.18) $k_{i j}(x)-k_{i j}(x-d)=O(1)$ for each fixed $d>0$.

(2.19) There exists $d>0$ such that

$$
\begin{gathered}
\liminf _{x \rightarrow \infty}\left[k_{i j}(x)-k_{i j}(x-d)\right]>0 ; i, j=1, \cdots, N . \\
\eta_{i j}(\delta)=\limsup _{x \rightarrow \infty}\left[k_{i j}(x)-k_{i j}(x-\delta)\right] \rightarrow 0 \text { as } \delta \rightarrow 0 .
\end{gathered}
$$


Definition 2.1. We let $k_{i j}^{(1)}(x)=k_{i j}(x)$ and

$$
k_{i j}^{(n+1)}(x)=\sum_{m=1}^{N} \int_{0}^{x} k_{i m}^{(n)}(x-y) d k_{m j}(y) .
$$

The next three results can be found in Pitt [1].

(2.21) Suppose $g(x) \sim a x^{n}$, then

$$
\int_{0}^{x} g(x-y) d k_{i j}(y) \sim \frac{a x^{n+1}}{N(n+1)} \quad \text { for all } i, j=1, \cdots, N .
$$

(2.22) For all pairs $(i, j) ; i, j=1, \cdots, N$ we have $k_{i j}^{(n)}(x) \sim x^{n} / N n !$.

(2.23) For all pairs $(i, j) ; i, j=1, \cdots, N$

$$
k_{i j}^{(n)}(x)-k_{i j}^{(n)}(x-d)=O\left(x^{n-1}\right) \text { for each fixed } d .
$$

LEMMA 2.3. For all $i, j=1, \cdots, N$ we have

$$
\underset{x \rightarrow \infty}{\limsup }\left|f_{i}(x)\right|=\limsup _{x \rightarrow \infty}\left|f_{j}(x)\right| \text {. }
$$

Proof. We let $S_{i}=\limsup \operatorname{sum}_{x \rightarrow \infty}\left|f_{i}(x)\right|$ and note that by Lemma 2.1 we have $\lim \sup _{x \rightarrow \infty}(1 / x) \int_{0}^{x}\left|f_{j}(x-y)\right| d k_{i j}(y) \leqq S_{j} / N$. From (2.15) it follows that $S_{i} \leqq N^{-1} \sum_{j=1}^{N} S_{j}$ for each $i=1, \cdots, N$. Suppose that for some $i_{0}$ we had $S_{i_{0}}<N^{-1} \sum_{j=1}^{N} S_{j}$, then summing on $i$ would give $\sum_{j=1}^{N} S_{j}<\sum_{j=1}^{N} S_{j}$. Hence $S_{i}=S_{j}=N^{-1} \sum_{m=1}^{N} S_{m}=S$.

LEMMA 2.4. For each $n$ we have

$$
\frac{S}{n !} \leqq \limsup _{x \rightarrow \infty} \sum_{j=1}^{N} \frac{1}{x^{n}} \int_{0}^{x}\left|f_{j}(x-y)\right| d k_{i j}^{(n)}(y) .
$$

Proof. By (2.15) the proposition is true for $n=1$. Suppose it is true for some $n \geqq 1$, i.e.,

$$
\frac{S}{n !} \leqq \limsup _{x \rightarrow \infty} \sum_{j=1}^{N} \frac{1}{x^{n}} \int_{0}^{x}\left|f_{j}(x-y)\right| d k_{i j}^{(n)}(y) .
$$

By (2.15) again,

$$
\begin{aligned}
\sum_{j=1}^{N} \frac{1}{x^{n}} & \int_{0}^{x}\left|f_{j}(x-y)\right| d k_{i j}^{(n)}(y) \\
& \leqq \sum_{j=1}^{N} \frac{1}{x^{n}} \int_{0}^{x}\left\{\sum_{m=1}^{N} \frac{1}{x-y} \int_{0}^{x-y}\left|f_{m}(x-y-t)\right| d k_{i m}(t)+o(1)\right\} d k_{i j}^{(n)}(y) \\
& \leqq \sum_{j, m=1}^{N} \frac{1}{x^{n}} \int_{0}^{x} \frac{1}{x-y} \int_{0}^{x-y}\left|f_{m}(x-y-t)\right| d k_{i m}(t) d k_{i j}^{(n)}(y)+o(1)
\end{aligned}
$$

by Lemma 2.1 . 
The first term equals

$$
\begin{aligned}
\sum_{j, m}^{N} & \frac{1}{x^{n+1}} \int_{0}^{x} \frac{x d k_{j}^{(n)}(y)}{x-y} \int_{0}^{x-y}\left|f_{m}(x-y-t)\right| d k_{j m}(t) \\
= & \sum_{j, m=1}^{N} \frac{1}{x^{n+1}} \int_{0}^{x} d k_{i j}^{(n)}(y) \int_{0}^{x-y}\left|f_{m}(x-y-t)\right| d k_{j m}(t) \\
& +\sum_{j, m=1}^{N} \frac{1}{x^{n+1}} \int_{0}^{x} \frac{y d k_{i j}^{(n)}(y)}{x-y} \int_{0}^{x-y}\left|f_{m}(x-y-t)\right| d k_{j m}(t) .
\end{aligned}
$$

The first term here is just $\sum_{m=1}^{N}\left(1 / x^{n+1}\right) \int_{0}^{x}\left|f_{m}(x-y)\right| d k_{i m}^{(n+1)}(y)$ by 2.12 . The lemma will be proved if we show that

$$
\underset{x \rightarrow \infty}{\limsup } \sum_{j, m=1}^{N} \frac{1}{x^{n+1}} \int_{0}^{x} \frac{y d k_{i j}^{(n)}(y)}{x-y} \int_{0}^{x-y}\left|f_{m}(x-y-t)\right| d k_{j m}(t) \leqq \frac{S}{n !}-\frac{S}{(n+1) !} .
$$

We let $g_{m}(x)=(1 / x) \int_{0}^{x}\left|f_{m}(x-y)\right| d k_{j m}(y)$ and note that $g_{m}(x)$ is bounded and $\lim \sup _{x \rightarrow \infty} g_{m}(x) \leqq S / N$.

The expression to be estimated is $\sum_{j, m=1}^{N}\left(1 / x^{n+1}\right) \int_{0}^{x} y g_{m}(x-y) d k_{j m}^{(n)}(y)$. Let $\varepsilon>0$ be given, then we choose $x_{0}$ so large that $g_{m}(t) \leqq S / N+\varepsilon$ for $t \geqq x_{0}$, then

$$
\begin{aligned}
& \frac{1}{x^{n+1}} \int_{0}^{x} y g_{m}(x-y) d k_{j m}^{(n)}(y) \\
& \quad=\frac{1}{x^{n+1}} \int_{0}^{x-x_{0}} y g_{m}(x-y) d k_{j m}^{(n)}(y)+\frac{1}{x^{n+1}} \int_{x-x_{0}}^{x} y g_{m}(x-y) d k_{j m}^{(n)}(y) .
\end{aligned}
$$

The second term is bounded by

$$
\frac{M}{x^{n}}\left[k_{i j}^{(n)}(x)-k_{i j}^{(n)}\left(x-x_{0}\right)\right]=O\left(\frac{1}{x}\right) \rightarrow 0,
$$

where $M$ is a bound for $g_{1}$, here we use (2.23).

The first term is bounded by

$$
\begin{aligned}
\left(\frac{S}{N}\right. & +\varepsilon) \frac{1}{x^{n+1}} \int_{0}^{x} y d k_{j m}^{(n)}(y) \\
& =\left(\frac{S}{N}+\varepsilon\right)\left[\frac{1}{x^{n}} \int_{0}^{x} d k_{j m}^{(n)}(y)-\frac{1}{x^{n+1}} \int_{0}^{x}(x-y) d k_{j m}^{(n)}(y)\right] \\
& =\left(\frac{S}{N}+\varepsilon\right)\left[\frac{k_{j m}^{(n)}(x)}{x^{n}}-\frac{1}{N(n+1) ! !}\right]+o(1) \\
& =\frac{1}{N}\left(\frac{S}{N}+\varepsilon\right)\left[\frac{1}{n !}-\frac{1}{(n+1) !}\right]+o(1) .
\end{aligned}
$$


Since $\varepsilon$ was arbitrary it follows that

$$
\underset{x \rightarrow \infty}{\lim \sup _{j, m}} \sum_{j, m} \frac{1}{x^{n+1}} \int_{0}^{x} y g_{m}(x-j) d k_{j m}(y) \leqq \frac{S}{n !}-\frac{S}{(n+1) !}
$$

which was to be proved.

We quote without proof the following elementary lemma, which can be found in Pitt [2].

(2.24) Suppose for $j=0,1, \cdots, J$ we have $q_{j}(x) \geqq 0$ for all $x$ and $q_{j}(x) \geqq 1$ in an interval $I_{j}$ of length $2 \alpha$ with center in a fixed interval $I$ of length $2 d$. Then there is an interval of length $2 \alpha$ with center in $I$, throughout which

$$
\sum_{j=0}^{J} q_{j}(x) \geqq \frac{J \alpha}{2(2 d+\alpha)}-1 \text {. }
$$

The next lemma is crucial and is based on an idea of Pitt.

LEMMA 2.5. If $\delta>0$ is given then there exist $x_{0}, n$, and $\beta>0$ such that for all $x \geqq x_{0}, k_{i j}^{(n)}(x)-k_{i j}^{(n)}(x-\delta) \geqq \beta x^{n-1}$, this holding for all pairs $(i, j)$.

Proof. Let $\phi_{i j}^{(n)}(x)=k_{i j}^{(n)}(x)-k_{i j}^{(n)}(x-\delta)$, then

$$
\begin{aligned}
\phi_{i j}^{(n+1)}(x) & =\sum_{m=1}^{N} \int_{0}^{x} k_{i m}^{(n)}(x-y) d k_{m j}(y)-\sum_{m=1}^{N} \int_{0}^{x-\delta} k_{i m}^{(n)}(x-\delta-y) d k_{m j}(y) \\
& =\sum_{m=1}^{N} \int_{0}^{x} \phi_{i m}^{(n)}(x-y) d k_{m j}(y) .
\end{aligned}
$$

The proof will be divided into several parts.

(2.5a) There exist $\alpha_{1}, \beta_{1}>0$ and $x_{1}$ such that for each pair $(i, j)$ there is a sequence of intervals $\left\{I_{m}(i, j)\right\}_{m=0}^{\infty}$ of length $2 \alpha_{1}$, such that every interval $(x, x+2 d)$ for $x \geqq x_{1}$ contains the center of one of these intervals and $\phi_{i j}^{(1)}(x) \geqq \beta_{1}$ for $x \in I_{m}(i, j)$. First by (2.19) choose $x_{1}^{\prime}$ so that $k_{i j}(x)-k_{i j}(x-d) \geqq \gamma>0$ for all $x \geqq x_{1}^{\prime}$. Then choose $\alpha$ so small that by (2.20)lim $\sup _{x \rightarrow \infty}\left[k_{i j}(x)-k_{i j}(x-\alpha)\right]<\gamma \delta / 4 d$, then choose $x_{1} \geqq x_{1}^{\prime}$ so that $k_{i j}(x)-k_{i j}(x-\alpha)<\gamma \delta / 4 d$ for all $x \geqq x_{1}$. Then it follows that for any $m$ such that $m d \geqq x_{1}$, the interval $(m d,(m+1) d)$ contains a subinterval of length $\alpha$, throughout which $\phi_{i j}^{(1)}(x) \geqq \gamma \delta / 4 d$. Clearly every interval of the type $(x, x+2 d)$ for $x \geqq x_{1}$ contains the center of such an interval. We can do this for each pair $(i, j)$ and hence can assume that $\alpha_{1}, \beta_{1}$, and $x_{1}$ are sufficient for all $(i, j)$. However the sequence of intervals $\left\{I_{m}(i, j)\right\}_{m=0}^{\infty}$ may vary with $(i, j)$.

(2.5b) There exists $h, \eta>0$ and $x_{2}$ such that for all $m$ such that $m d \geqq x_{2}$, and for each $(i, j)$ the interval $(m d,(m+1) d)$ contains 2 subintervals $\left(m d+a_{m}(i, j)\right.$, $\left.m d+a_{m}(i, j)+h\right)$ and $\left(m d+b_{m}(i, j), m d+b_{m}(i, j)+h\right)$ such that $k_{i j}$ increases by at least $\eta$ in each of these intervals and:

$$
b_{m}(i, j)-a_{m}(i, j)+h \leqq 2 \alpha_{1}
$$




$$
a_{m}(i, j)+2 h \leqq b_{m}(i, j) .
$$

First choose $x_{2}^{\prime}$ so that for all $m$ such that $m d \geqq x_{2}^{\prime}, k_{i j}$ increases by more than $\gamma^{\prime}>0$ in the interval from $m d$ to $(m+1) d$. In any such interval clearly there is a subinterval of length $2 \alpha_{1}$, throughout which $k_{i j}$ increases by at least $\gamma>0$, we may take $\gamma=\gamma^{\prime} 2 \alpha_{1} / d$ and we see that $\gamma$ is independent of $m$. Now choose $h$ so small that $\lim \sup _{x \rightarrow \infty}\left[k_{i j}(x)-k_{i j}(x-h)\right]<\gamma / 4$ and then $x_{2} \geqq x_{2}^{\prime}$ so that

$$
k_{i j}(x)-k_{i j}(x-h)<\frac{\gamma}{4} \quad \text { for all } x \geqq x_{2} .
$$

Now let $\eta=\gamma h / 8 d$. Let $m$ be such that $m d \geqq x_{2}$, then $(m d,(m+1) d)$ contains a subinterval of length $2 \alpha_{1}$ such that $k_{i j}$ increases by at least $\gamma$ in this subinterval. We divide this subinterval into subintervals of length $h$, in at least one of these $k_{i j}$ increases by more than $\eta$, pick the first one, then skip one and there must be at least one more farther on where it increases by at least $\eta$. Let $a_{m}(i, j), b_{m}(i, j)$ be the left-hand end points of these intervals, then conditions (2.25), (2.26) follow by the construction.

(2.6c) Proceed by induction. We assume that for some $n \geqq 1$, there exists $\beta_{n}>0, \alpha_{n} \geqq \alpha_{1}+(n-1 / 2) h$, and $x_{n}$ such that for each $(i, j)$ there is a sequence $\left\{I_{m}^{(n)}(i, j)\right\}_{m=0}^{\infty}$ of intervals of length $2 \alpha_{n}$, such that for $x \geqq x_{n}$ the interval $(x, x+2 d)$ contains the center of one of these intervals and for $x \in I_{m}^{(n)}(i, j)$, $\phi_{i j}^{(n)}(x) \geqq \beta_{n} x^{n-1}$. We have already verified this for $n=1$.

Fix $(i, j)$; we have

$$
\begin{aligned}
\phi_{i j}^{(n+1)} & =\sum_{m=1}^{N} \int_{0}^{x} \phi_{i m}^{(n)}(x-y) d k_{m j}(y) \\
& =\sum_{r=1}^{N} \sum_{m \geqq 0} q_{r, m}(x),
\end{aligned}
$$

where

$$
q_{r, m}(x)=\int_{m d}^{(m+1) d} \phi_{i r}^{(n)}(x-y) d k_{r j}(y) .
$$

Now choose $m_{0}$ so that $m d \geqq x_{2}$ for $m \geqq m_{0}$ and pick $X \geqq x_{n}, X \geqq(m+1) d$. By the induction hypothesis, for each $(r, j)$ there exists $\tau_{r} \in(X, X+2 d)$ such that $\phi_{i r}^{(n)}(y) \geqq \beta_{n} y^{n-1}$ for $y \in\left(\tau_{r}-\alpha_{n}, \tau_{r}+\alpha_{n}\right)$.

Now suppose $x$ is in one of the intervals

$$
\begin{aligned}
& I_{1}=\left(\tau_{r}-\alpha_{n}+m d+a_{m}(r, j)+h, \tau_{r}+\alpha_{n}+m d+a_{m}(r, j)\right), \\
& I_{2}=\left(\tau_{r}-\alpha_{n}+m d+b_{m}(r, j)+h, \tau_{r}+\alpha_{n}+m d+b_{m}(r, j)\right) .
\end{aligned}
$$


If $x \in I_{1}$, then

$$
\begin{aligned}
q_{r, m}(x) & =\int_{m d}^{(m+1) d} \phi_{i r}^{(n)}(x-y) d k_{r j}(y) \\
& \geqq \int_{m d+a_{m}(r, j)}^{m d+a_{m}(r, j)+h} \phi_{i r}^{(n)}(x-y) d k_{r j}(y) \\
& \geqq \int_{m d+a_{m}(r, j)}^{m d+a_{m}(r, j)+h} \beta_{u}(x-y)^{n-1} d k_{r j}(y) \\
& \geqq \eta \beta_{n}(x-m d-d)^{n-1},
\end{aligned}
$$

because if $x \in I_{1}$ and $y \in\left(m d+a_{m}(r, j), m d+a_{m}(r, j)+h\right)$ then

$$
\tau_{r}-\alpha_{n} \leqq x-y \leqq \tau_{r}+\alpha_{n} .
$$

Similarly, if $x \in I_{2}$, then

$$
\begin{aligned}
q_{r, m}(x) & \geqq \int_{m d+b_{m}(r, j)}^{m d+b_{m}(r, j)+h} \phi_{\iota r}^{(n)}(x-y) d k_{r j}(y) \\
& \geqq \eta \beta_{n}(x-m d-d)^{n-1} .
\end{aligned}
$$

By (2.25), (2.26) these intervals overlap and have total length of at least $2 \alpha_{n}+h$.

Now consider some large $X \geqq x_{n}$.

$$
\begin{aligned}
\phi_{i j}^{(n+1)}(x) & =\sum_{r=1}^{N} \sum_{m \geqq 0} q_{r, m}(x) \\
& \geqq \sum_{r=1}^{N} \sum_{m_{0} \leqq m \leqq X / 2 d-1} q_{r, m}(x) .
\end{aligned}
$$

Welfocus our attention on one of the $q_{r, m}$ appearing in this sum. Now $X-m d>m$ so there exists $\tau_{r} \in(X-m d, X-(m-1) d)$ such that $\phi_{i r}^{(n)}(y) \geqq \beta_{n} y^{n-1}$ for $y \in\left(\tau_{r}-\alpha_{n}, \tau_{r}+\alpha_{n}\right)$. One easily checks that the center of the corresponding interval $I_{1} \cup I_{2}$ lies in $(X, X+2 d)$. So we see from the above that for each of these functions $q_{r, m}$ with $r=1, \cdots, N$ and $m_{0} \leqq m \leqq X / 2 d-1$, the interval $(X, X+2 d)$ contains the center of an interval of length $2 \alpha_{n}+h$ throughout which

$$
q_{r, m} \geqq \eta \beta_{n}(x-m d-d)^{n-1} \geqq \eta \beta_{n}\left(\frac{X}{2}\right)^{n-1},
$$

since $(m+1) d \geqq X / 2$. Therefore by (2.24) this interval contains the center of an interval of length $2 \alpha_{n}+h$, throughout which

$$
\phi_{i j}^{(n+1)}(x) \geqq \eta \beta_{n}\left(\frac{X}{2}\right)^{n-1}\left\{\frac{N\left(\left(\frac{X}{2 d}\right)-1-m_{0}\right)\left(\alpha_{n}+\frac{h}{2}\right)}{2\left(2 d+\alpha_{n}+\frac{h}{2}\right)}-1\right\} \geqq \beta_{n+1}^{\prime} X^{n}
$$


if $X$ is taken sufficiently large. But since $X \geqq x-4 d$ for all $x$ in this interval we have $\phi_{i j}^{(n+1)}(x) \geqq \beta_{n} x^{n}$ for all $X$ sufficiently large. So we have verified the proposition for $n+1$. Now we just take $n$ so large that $2 \alpha+(n-1) h>4 d$ and then we will have

$$
\phi_{i j}^{(n)}(x) \geqq \beta_{n} x^{n-1} \quad \text { for all large } x .
$$

THEOREM 2.1. For each $i=1, \cdots, N, f_{i}(x)=o(1)$.

Proof. We want to show $S=0$. Suppose $S>0$. Then by Lemma 2.2 there exist $x_{0}, T, \delta$ such that, for all $x \geqq x_{0}$, the interval $(x, x+T)$ contains a subinterval of length $\delta$, throughout which $\left|f_{i}(t)\right|<S / 2$ for all $i=1, \cdots, N$. Now choose $x_{1}$ and $n$ so that for all $(i, j)$

$$
k_{i j}^{(n)}(x)-k_{i j}^{(n)}(x-\delta) \geqq \beta x^{n-1}
$$

for some $\beta>0$ and all $x \geqq x_{1}$. This is possible by Lemma 2.5. Let $x_{2}=\max \left(x_{1}, x_{2}\right)$. Suppose $x-T \geqq x_{2}$, and $(y-\delta, y) \subseteq(x-T, x)$. Then $k_{i j}^{(n)}(y)-k_{i j}^{(n)}(y-\delta) \geqq \beta x^{n-1}$ and $k_{i j}^{(n)}(x)-k_{i j}^{(x)}(x-T) \leqq A x^{n-1}$ by (2.23). Hence

$$
\frac{k_{i j}^{(n)}(y)-k_{i j}^{(n)}(y-\delta)}{k_{i j}^{(n)}(x)-k_{i j}^{(n)}(x-T)} \geqq \frac{\beta}{A},
$$

or $k_{i j}^{(n)}(y)-k_{i j}^{(n)}(y-\delta)>\gamma\left[k_{i j}^{(n)}(x)-k_{i j}^{(n)}(x-T)\right]$ for some $\gamma>0$. Now Lemma 2.4 gives

$$
\frac{S}{n !} \leqq \limsup _{x \rightarrow \infty} \sum_{j=1}^{N} \frac{1}{x^{n}} \int_{0}^{x}\left|f_{j}(x-y)\right| d k_{i j}^{(n)}(y)
$$

for each $i=1, \cdots, N$. In order to get a contradiction it is enough to show that

$$
\limsup _{x \rightarrow \infty} \frac{1}{x^{n}} \int_{0}^{x}\left|f_{j}(x-y)\right| d k_{i j}^{(n)}(y)<\frac{S}{N n !} .
$$

Let $\varepsilon>0$ be given, we assume that $x_{2}$ chosen before is also so large that $\left|f_{j}(t)\right|<S+\varepsilon$ for all $t \geqq x_{2}$. Now

$$
\begin{aligned}
& \frac{1}{x^{n}} \int_{0}^{x}\left|f_{j}(x-y)\right| d k_{i j}^{(n)}(y) \\
& \quad=\frac{1}{x^{n}} \int_{0}^{x-x_{2}}\left|f_{j}(x-y)\right| d k_{i j}^{(n)}(y)+\frac{1}{x^{n}} \int_{x-x_{2}}^{x}\left|f_{j}(x-y)\right| d k_{i j}^{(n)}(y)
\end{aligned}
$$

The second integral goes to zero by (2.23) and the fact that $f_{j}$ is bounded. The first term is broken up as follows: 


$$
\begin{aligned}
& \sum_{m=0}^{\left[\left(x-x_{2}\right) / T-1\right]} \frac{1}{x^{n}} \int_{m T}^{(m+1) T}\left|f_{j}(x-y)\right| d k_{i j}^{(n)}(y) \\
& \leqq \sum_{m=0}^{\left[\left(x-x_{2}\right) / T-1\right]} \frac{1}{x^{n}}\left\{(S+\varepsilon)\left[k_{i j}^{(n)}((m+1) T)-k_{i j}^{(n)}(m T)\right]-\frac{S}{2}\left[k_{i j}^{(n)}\left(y_{m}+\delta\right)-k_{i j}^{(n)}\left(y_{m}\right)\right]\right\} \\
& \leqq \sum_{m=0}^{\left[\left(x-x_{2}\right) / T-1\right]} \frac{1}{x^{n}}\left\{(S+\varepsilon)-\gamma \frac{S}{2}\right\}\left[k_{i j}^{(n)}((m+1) T)-k_{i j}^{(n)}(m T)\right] \\
& =\frac{1}{x^{n}}\left\{(S+\varepsilon)-\gamma \frac{S}{2}\right\} k_{i j}^{(n)}\left(x-x_{2}\right) .
\end{aligned}
$$

Hence $\limsup \operatorname{sum}_{x \rightarrow \infty}\left(1 / x^{n}\right) \int_{0}^{x}\left|f_{j}(x-y)\right| d k_{i j}^{(n)}(y) \leqq S(1-\gamma / 2) / N n$ ! since $\varepsilon$ was arbitrary. This gives a contradiction and hence $S=0$.

COROLlaRy 2.1. If the $f_{i}(x)$ are permitted to take on complex values with the restriction that there exist $\alpha_{i}, i=1, \cdots, N$ such that $\alpha_{i} \leqq \arg f_{i}(x) \leqq \alpha_{i}+\pi$ then Theorem 2.1 still holds.

Proof. Noting that $e^{-\imath \alpha_{j}} f_{j}(x)$ satisfies (2.13)-(2.16) whenever $f_{j}(x)$ does we may assume that $0 \leqq \arg f_{j}(x) \leqq \pi$ for $j=1, \cdots, N$. Let $f_{j}(x)=f_{j}^{(1)}(x)+i f_{j}^{(2)}(x)$. Then we see that $f_{j}^{(2)}(x) \geqq 0$ for all $x$. By condition (2.14), $\int_{0}^{x} f_{j}^{(2)}(t) d t=O(1)$, hence $\int_{0}^{\infty} f_{j}^{(2)}(t) d t$ exists because $f_{j}^{(2)}(t) \geqq 0$. Since $f_{j}$ satisfies $(2.16)$ so does $f_{j}^{(2)}$ and it follows easily from this and the fact that $\int_{0}^{\infty} f_{j}^{(2)}(t) d t<\infty$, that $f_{j}^{(2)}(x)=o(1)$. By (2.15) we have

$$
\left|f_{j}(x)\right| \leqq \sum_{m=1}^{N} \frac{1}{x} \int_{0}^{x}\left|f_{m}(x-y)\right| d k_{j m}(y)+o(1) .
$$

Now $\left|f_{j}(x)\right|=\left|f_{j}^{(1)}(x)\right|+o(1)$ and

$$
\begin{aligned}
\frac{1}{x} \int_{0}^{x}\left|f_{m}^{(1)}(x-y)+i f_{m}^{(2)}(x-y)\right| d k_{j m}(y) & \\
& \leqq \frac{1}{x} \int_{0}^{x}\left|f_{m}^{(1)}(x-y)\right| d k_{j m}(y)+\frac{1}{x} \int_{0}^{x}\left|f_{m}^{(2)}(x-y)\right| d k_{j m}(y) .
\end{aligned}
$$

The second integral is $o(1)$, by Lemma 2.1. Hence

$$
\left|f_{j}^{(1)}(x)\right| \leqq \sum_{m=1}^{N} \frac{1}{x} \int_{0}^{x}\left|f_{m}^{(1)}(x-y)\right| d k_{j m}(y)+o(1)
$$

for $j=1, \cdots, N$. Since $f_{j}^{(1)}(x)$, for $j=1, \cdots, N$ satisfy (2.13)-(2.16) it follows from Theorem 2.1 that $f_{j}^{(1)}(x)=o(1)$, hence $f_{j}(x)=o(1)$.

Next we show that given any angular sector in the plane: $\alpha \leqq \theta \leqq \pi+\alpha+\varepsilon$ for $\varepsilon>0$, there exists a function $f$ taking on values in this sector such that $f$ satisfies conditions (2.13), (2.14), (2.15) and that for any monotone increasing function $k$, with $k(x) \sim x$ we have 


$$
|f(x)| \leqq \frac{1}{x} \int_{0}^{x}|f(x-y)| d k(y)+o(1) ; \text { yet } f(x) \neq o(1) .
$$

We may assume the angular sector is of the type $-\varepsilon \leqq \theta \leqq \pi+\varepsilon$, for some $\varepsilon>0$. We define $f$ as follows:

$$
\begin{aligned}
& f(t)=e^{t(\pi / 2-t)} \quad \text { for } 0 \leqq t \leqq \pi / 2+\varepsilon, \\
& f(t)=e^{-i \varepsilon} \quad \text { for } \pi / 2+\varepsilon \leqq t \leqq T, \text { where } T=\pi / 2+\varepsilon+\cot \varepsilon, \\
& f(t)=f(2 t-T) \quad \text { for } T \leqq t \leqq 2 T, \\
& f(t)=-\overline{f(t-2 T)} \text { for } 2 T \leqq t \leqq 4 T,
\end{aligned}
$$

then define $f$ periodically with period $4 T$.

It is easily seen that $f$ is continuous, and hence it is uniformly continuous since it is periodic. Therefore it satisfies (2.16). Since $|f(t)|=1$, condition (2.13) is fulfilled.

To show that (2.14) is satisfied we show that $\int_{0}^{4 T} f(t) d t=0$. Let $f(t)=f_{1}(t)+i f_{2}(\mathrm{t})$,

$$
\begin{aligned}
\int_{2 T}^{4 T} f_{1}(t) d t & =\int_{2 T}^{4 T} R\{-\overline{f(t-2 T)\}} d t \\
& =-\int_{2 T}^{4 T} f_{1}(t-2 T) d t=-\int_{0}^{2 T} f_{1}(t) d t
\end{aligned}
$$

hence $\int_{0}^{4 T} f_{1}(t) d t=0$. Next consider $f_{2}(t)$,

$$
\begin{aligned}
\int_{0}^{T} f_{2}(t) d t & =\int_{0}^{\pi / 2+\varepsilon} \sin (\pi / 2-t) d t-\sin \varepsilon \int_{\pi / 2+\varepsilon}^{T} d t \\
& =\int_{0}^{\pi / 2+\varepsilon} \cos t d t-\cot \varepsilon \sin \varepsilon \\
& =\sin (\pi / 2+\varepsilon)-\cos \varepsilon=0 .
\end{aligned}
$$

Furthermore,

hence

$$
\int_{T}^{2 T} f_{2}(t) d t=\int_{T}^{2 T} f_{2}(2 T-t) d t=\int_{0}^{T} f_{2}(t) d t=0,
$$

$$
\int_{0}^{2 T} f_{2}(t) d t=0
$$

Next:

$$
\int_{2 T}^{4 T} f_{2}(t) d t=\int_{2 T}^{4 T} \operatorname{Im}\{-\overline{f(t-2 T)}\} d t=\int_{2 T}^{4 T} f_{2}(t-2 T) d t=\int_{0}^{2 T} f_{2}(t) d t=0,
$$

so

$$
\int_{0}^{4 T} f_{2}(t) d t=0
$$


Since $|f(t)|=1$, the condition that $|f(x)| \leqq(1 / x) \int_{0}^{x} f(x-y) d k(y)+o(1)$ merely requires that $1 \leqq k(x) / x+o(1)$ which is true for any $k$ such that $k(x) \sim x$.

Finally, (2.9) can be written as:

$$
\left|F_{h}(x)\right| \leqq \sum_{g \in G_{\mathfrak{m}}} \frac{1}{x} \int_{0}^{x}\left|F_{g}(x-y)\right| d H_{h, g}(y)+o(1)
$$

for all $h \in G_{\mathfrak{m}}$, where $F_{h}(x)=f_{h}\left(e^{x}\right)$ and $H_{h, g}(y)=\sum_{n \leqq e^{y}} \beta_{h g}(n)$. We point out that if $f(x)$ is given by (2.2) or (2.4) and $\alpha(\mathfrak{a})=\Lambda(\mathfrak{a}) / N \mathfrak{a}$, it is possible to show by elementary methods that the conditions of Theorem 2.1 are satisfied.

\section{BIBLIOGRAPHY}

S. A. Amrtsur

1. On arithmetic functions, J. Analyse Math. 5 (1956/57), 273-314.

2. Some results on arithmetic functions, J. Math. Soc. Japan 2 (1959), 275-290.

E. LANDAU

1. Uber Ideale und Primideale in Idealklassen, Math. Z. 2 (1918), 52-159.

H. R. PITT

1. A general Tauberian theorem related to the elementary proof of the prime number theorem, Proc. London Math. Soc. (3) 8 (1958), 569-588.

2. Tauberian theorems, Oxford, 1958.

A. Selberg

1. An elementary proof of the prime number theorem, Ann. of Math. (2) 50 (1949), 305-313.

2. An elementary proof of the prime number theorem for arithmetic progressions, Canad. J. Math. 2 (1950), 66-78.

H. N. SHAPIRo and W. Forman

1. Abstract prime number theorems, Comm. Pure Appl. Math. 7 (1954), 587-619.

The Institute for Advanced Study,

Princeton, NeW Jersey 